\title{
Diagnóstico de hanseníase em Porto Nacional/TO no período de 2013 a 2017
}

A hanseníase é uma doença crônica e insidiosa cujo agente etiológico é a bactéria Mycobacterium leprae, de elevada infectividade, porém de baixa patogenicidade. Sua transmissão ocorre pelo ar, liberado por indivíduos contaminados e infectando indivíduos susceptíveis. As manifestações clínicas, geralmente, demoram a aparecer, levando a um diagnóstico tardio sendo que, nesse período, o infectado é o transmissor da doença. O tratamento se faz por meio de poliquimioterapia por um tempo prolongado, que ocorre, principalmente, na unidade básica de saúde. O objetivo do presente trabalho é avaliar os dados estatísticos de diagnóstico de hanseníase no município de Porto Nacional/TO entre o período de 2013 a 2017. Trata-se de um estudo descritivo de investigação epidemiológica e revisão de literatura de dados presentes no SINAN (Sistema Nacional de Agravos de Notificação) juntamente à Secretaria de Vigilância em Saúde, dados do banco de informações do DataSUS e levantamentos bibliográficos eletrônicos de bibliotecas virtuais. Foi observado grande variação de diagnóstico de hanseníase entre o período de estudo, prevalência da forma multibacilar, prevalência de diagnóstico de hanseníase sem acometimento do tronco nervoso e predisposição ao sexo masculino. Os níveis de diagnósticos permaneceram variáveis, com maioria masculino, de forma multibacilar, sendo 12 recidivas.

Palavras-chave: Hanseníase; Hanseníase multibacilar; Mycobacterium leprae.

\section{Diagnosis of leprosy in Porto Nacional/TO between 2013 to 2017}

The leprosy is a chronic and insidious disease whose etiological agent is Mycobacterium leprae, a highly infectious but low pathogenic bacterium. Its transmission occurs through the air, released by contaminated individuals and infecting susceptible individuals. The clinical manifestations usually delay to appear, leading to a late diagnosis and, in that period, the infected is the transmitter of the disease. The treatment is done by means of polychemotherapy, for a prolonged time, that occurs, mainly, in the basic health unit. The objective of the present study is to evaluate the statistical data of leprosy diagnosis in the municipality of Porto Nacional/TO between the period of 2013 to 2017. This is a descriptive study of epidemiological investigation and literature review of data present in SINAN (National System of Notification Aggravations) together with the Health Surveillance Secretariat, DataSUS information bank data and electronic bibliographic surveys of virtual libraries. There was big variation of leprosy diagnosis between the study period, prevalence of the multibacillary form, prevalence of leprosy diagnosis without involvement of the nervous trunk and predisposition to the male sex. The diagnostic levels remained variable, with a masculine majority, in a multibacillary form, with 12 recurrences.

Keywords: Leprosy; Multibacillary lepra; Mycobacterium leprae.

Topic: Epidemiologia

Reviewed anonymously in the process of blind peer.
Received: 10/06/2020

Approved: $19 / 08 / 2020$

Felipe Alior Fernandes Louzada de Almeida

Faculdade Presidente Antônio Carlos, Brasil

http://lattes.cnpq.br/2909152614160765

http://orcid.org/0000-0001-9724-9256

felipe-alior@hotmail.com

\section{George Milan (id}

Faculdade Presidente Antônio Carlos, Brasil

http://lattes.cnpq.br/0566763711448875

http://orcid.org/0000-0002-1506-0132

geomilan@hotmail.com

\section{Referencing this:}

ALMEIDA, F. A. F. L.; MILAN, G.. Diagnóstico de hanseníase em Porto Nacional/TO no período de 2013 a 2017. Scire Salutis, v.10, n.3, p.104112, 2020. DOI: http://doi.org/10.6008/CBPC22369600.2020.003.0013 


\section{INTRODUÇÃO}

A hanseníase (CID: A30), conhecida também como doença da Hansen, ou ainda como lepra, é uma infecção crônica, granulomatosa, curável, que tem como agente etiológico a bactéria Mycobacterium leprae (bacilo de Hansen) (WHITE et al., 2015). É um micro-organismo de elevada infectividade, porém de baixa patogenicidade, ou seja, muitos indivíduos entram em contato com a bactéria, porém poucos adoecem (GRACIE, 2017), atingindo pessoas de todas as idades e sexos, entretanto é raro em crianças abaixo de 5 anos e idosos. É considerada um dos principais problemas de saúde no mundo, especialmente no Brasil, um dos poucos países que ainda não atingiu a meta de erradicação completa da doença, considerado o segundo país mais endêmico do mundo, evidenciando dificuldade da saúde pública em controlar a infecção (LUNA et al., 2018).

Nos últimos anos vários foram os programas de saúde, apoiado pela Organização Mundial de Saúde (OMS), para o seu controle, detecção precoce da hanseníase e tratamento gratuito com poliquimioterapia (PQT) (VELOSO, 2018), como a "Estratégia Global para Maior Redução da Carga da Hanseníase e a Sustentação das Atividades de Controle da Hanseníase: 2006-2010", Estratégia Global Aprimorada para Redução Adicional da Carga da Hanseníase: 2011-2015, e por último, Campanha Nacional de hanseníase, geo-helmintiases e Tracoma. Só em 2014 foram registrados 213 mil casos novos mundialmente, sendo o Brasil responsável por 36.521 casos, aproximadamente, e por 93\% dos casos de hanseníase em todo o continente americano (MONTEIRO et al., 2015). Os estados em que prevalecem o coeficiente de Hanseníase são: Mato Grosso, Tocantins, Maranhão, Rondônia e Pará (FREITAS et al., 2017).

A transmissão da bactéria ocorre pelo ar, no qual o infectado sem tratamento (homem) libera os patógenos através das vias aéreas superiores até atingir o indivíduo susceptível (MONTEIRO et al., 2017). O agente patológico tem um tropismo para o Sistema Nervoso Periférico (SNP), levando a lesões de origem sensitiva-dolorosa da pele (GIRÃO NETA et al., 2017). Entretanto, vários fatores predispõem para que o indivíduo seja infectado e torna-se sintomático, são eles: predisposição genética, imunidade e virulência da bactéria (FORTUNA et al., 2018).

Existem alguns modos para classificar a hanseníase. O mais utilizado no Brasil é a classificação operacional da OMS que divide basicamente em dois grupos: Paucibacilar (PB) e Multibacilar (MB) (PEIXOTO et al., 2016), que auxilia também no tratamento. O grupo PB é caracterizado nos indivíduos com menos de 5 lesões na pele, o que reflete a baixa carga de bacilos no organismo impedindo-o de transmitir a outros indivíduos. Já no grupo $\mathrm{MB}$, os indivíduos apresentam mais de cinco lesões na pele ou apresentam lesão neural, como espessamento dos nervos (FORTUNA et al., 2018).

A manifestação clínica leva em conta, principalmente, a resposta imune do hospedeiro. Porém o que é possível ver em todos os tipos, e o que define a doença, são as manchas hipocrômicas localizadas na epiderme, derme, podendo alcançar até o tecido adiposo, associado a alterações de sensibilidade (anestesia e anidrótica), manifestações sistêmicas, como queda de pelos, especialmente da sobrancelha, pápulas, infiltrações, tubérculos e nódulos sem quaisquer sintomas associados (RODRIGUES, 2016). 
Apesar do quadro clínico ser vasto, há diferentes modos de manifestar a hanseníase. Sendo assim, utiliza-se a Classificação de Madri, feito no Congresso de hanseníase em 1953, aceito até hoje, que classifica o hansênico em 04 (quatro) tipos de acordo com o exame físico: virchowiano (forma multibacilar, caracterizada por infiltração progressiva da pele, acometendo órgãos como olhos, testículos, linfonodos e baço); forma tuberculoide (lesões hipocrômicas que apresentam em suas bordas placas com bordas papulosas e eritematosas); indeterminado (caracterizado por manchas hipocrômicas, de bordas imprecisas que se localizam em qualquer região da pele, sem comprometer ramos nervosos); e por fim, a forma dimorfa (estão em indivíduos com instabilidade no sistema imune, tendo grandes variações nas manifestações clínicas, desde lesões neurais assimétricas quanto comprometimento sistêmico) (FORTUNA et al., 2018).

O diagnóstico é essencialmente clínico e epidemiológico. Não há teste laboratorial sensível e específico para concretizar a hanseníase (RODRIGUES, 2016). As lesões suspeitas de hanseníase devem ser estudadas por meio de exame físico dermatológico e neural, por profissionais aptos a realizar as condutas, por ser uma doença peculiar, no qual há um exame clínico específico (VELOSO, 2018). Na maioria dos casos, a pesquisa das lesões é realizada a nível de atenção básica de saúde, com o objetivo de avaliar lesões hipocrômicas, alterações de sensibilidade e comprometimento do SNP. Nesse momento, é feito a classificação quanto a PB ou MB, e o modo de apresentação da doença (AMORIM et al., 2016).

O tratamento é feito por um conjunto de antibióticos denominado poliquimioterapia (PQT), com o intuito de eliminar a bactéria do organismo (LIRA et al., 2017), adotado pela OMS a nível mundial. Após o diagnóstico, deve-se classificar o paciente hansênico para iniciar o esquema de terapia específico para o indivíduo (FORTUNA et al., 2018). Dessa forma, para os PB utiliza-se o esquema de rifampicina, uma dose mensal de $600 \mathrm{mg}$, supervisionado na atenção básica de saúde, dapsona, uma dose mensal supervisionada e uma dose diária autoadministrada. Nesse caso, o tratamento dura 6 meses. Já a MB utiliza as mesmas doses da PB associando a clofazimina, uma dose mensal de $300 \mathrm{mg}$ com administração supervisionada e uma dose diária de 50 mg autoadministrada, sendo a duração do tratamento de 12 meses (MONTALVÃO, 2018).

Diante do exposto, o presente estudo tem por objetivo geral analisar os números de casos notificados de hanseníase pelo Sistema de Informação de Agravos de Notificação (SINAN) no período de 01 de janeiro de 2013 a 31 de dezembro de 2017, visto que a OMS iniciou um programa em 2014 para diminuir os casos de hanseníase no Brasil até 2020. O objetivo do presente trabalho é avaliar os dados estatísticos de diagnóstico de hanseníase no município de Porto Nacional/TO entre o período de 2013 a 2017.

\section{METODOLOGIA}

O presente estudo trata-se de uma pesquisa descritiva epidemiológica com investigação bibliográfica, de caráter quantitativo. Os dados foram obtidos no SINAN, um site que facilita a avaliação dos planos e projetos de saúde, melhorando a tomada de decisões e transmitir os dados aos profissionais de saúde. Também foi disponibilizado informações do banco de informações do DataSUS, que serve para subsidiar análises e evidências quanto à colaboração dos programas de saúde.

A coleta de dados foi feita durante o período de 24 de setembro de 2018 até 10 de outubro de 2018. 
Além disso, foram captadas referências bibliográficas no período de 2014 a 2018, onde foi buscado programas da OMS e dados epidemiológicos. Para melhor avaliação dos dados, estes foram tabelados em uma planilha Excel 2010, expressos em números e porcentagens e, posteriormente, em gráficos. As variáveis foram coletadas pelos pesquisadores autores deste projeto. A idade não foi definida, os indivíduos que receberam o diagnóstico de hanseníase deveriam ser do município de Porto Nacional, independentemente de ser zona urbana ou rural, e o sexo dividido em feminino e masculino.

\section{RESULTADOS}

De acordo com os levantamentos de dados feito de pacientes infectados pela hanseníase, observou um maior número de casos de hanseníase em homens nos 4 anos estudados, como mostra a tabela 1. Dos 247 casos notificados no município de Porto Nacional/TO, 154 foram do sexo masculino (62,34\%) e 93, feminino $(37,65 \%)$.

Tabela 1: Frequência de infecção por hanseníase de acordo com o gênero.

\begin{tabular}{lllllll}
\hline Sexo & $\mathbf{2 0 1 3}$ & $\mathbf{2 0 1 4}$ & $\mathbf{2 0 1 5}$ & $\mathbf{2 0 1 6}$ & $\mathbf{2 0 1 7}$ & Total \\
\hline Masc & 29 & 35 & 29 & 30 & 31 & 154 \\
\hline Fem & 15 & 24 & 12 & 20 & 22 & 93 \\
\hline Total & 44 & 59 & 41 & 50 & 53 & 247
\end{tabular}

Fonte: Elaborada com dados da Vigilância Epidemiológica de Porto Nacional/TO.

No período de 2013 a 2017 os casos de hanseníase houve significativa alteração no que tange ao número total de casos, com uma média de 49,5 casos notificados/ano. As maiores frequências foram vistas no ano de 2014 , como mostra a tabela 2, a seguir.

Tabela 2: Frequência quanto ao número de casos notificados de hanseníase no município de Porto Nacional/TO por ano.

\begin{tabular}{|c|c|c|c|c|c|c|}
\hline Mês da Notificação & 2013 & 2014 & 2015 & 2016 & 2017 & Total \\
\hline Total & 44 & 59 & 41 & 50 & 53 & 247 \\
\hline Jan & 2 & 1 & 4 & 4 & 2 & 13 \\
\hline Fev & 1 & 5 & 1 & 2 & 4 & 13 \\
\hline Mar & 7 & 2 & 5 & 4 & 8 & 26 \\
\hline Abr & 4 & 7 & 3 & 7 & 1 & 22 \\
\hline Maio & 6 & 5 & 0 & 2 & 12 & 25 \\
\hline Jun & 2 & 6 & 7 & 6 & 9 & 30 \\
\hline Jul & 5 & 6 & 1 & 1 & 1 & 14 \\
\hline Agos & 7 & 5 & 3 & 6 & 0 & 21 \\
\hline Set & 3 & 5 & 0 & 0 & 3 & 11 \\
\hline Out & 1 & 6 & 11 & 7 & 6 & 31 \\
\hline Nov & 5 & 6 & 5 & 6 & 4 & 26 \\
\hline Dez & 1 & 5 & 1 & 5 & 3 & 15 \\
\hline
\end{tabular}

Fonte: Elaborada com dados da Vigilância Epidemiológica de Porto Nacional/TO.

No tocante ao número de nervos acometidos, percebidos pelo exame físico, avaliou-se um predomínio de 0 nervos acometidos, correspondendo a 33,19\% (82 casos notificados), 71 indivíduos tiveram entre 1 a 3 nervos acometidos; 64, entre 4 a 6 nervos acometidos; 27 , entre 7 a 9 nervos acometidos e apenas 3 tiveram mais de 10 nervos acometidos, como é mostrado no gráfico 1.

A distribuição dos casos foi realizada de segundo a classificação de Madri (forma indeterminada, Tuberculóide, Dimorfa e Virchowiana), baseando-se nas características da forma clínica da doença. Por outro 
lado, a classificação do OMS, que é muito mais utilizada no Brasil por ela ser preditivo para a melhor escolha medicamentosa, obteve os resultados segundo a tabela 4.

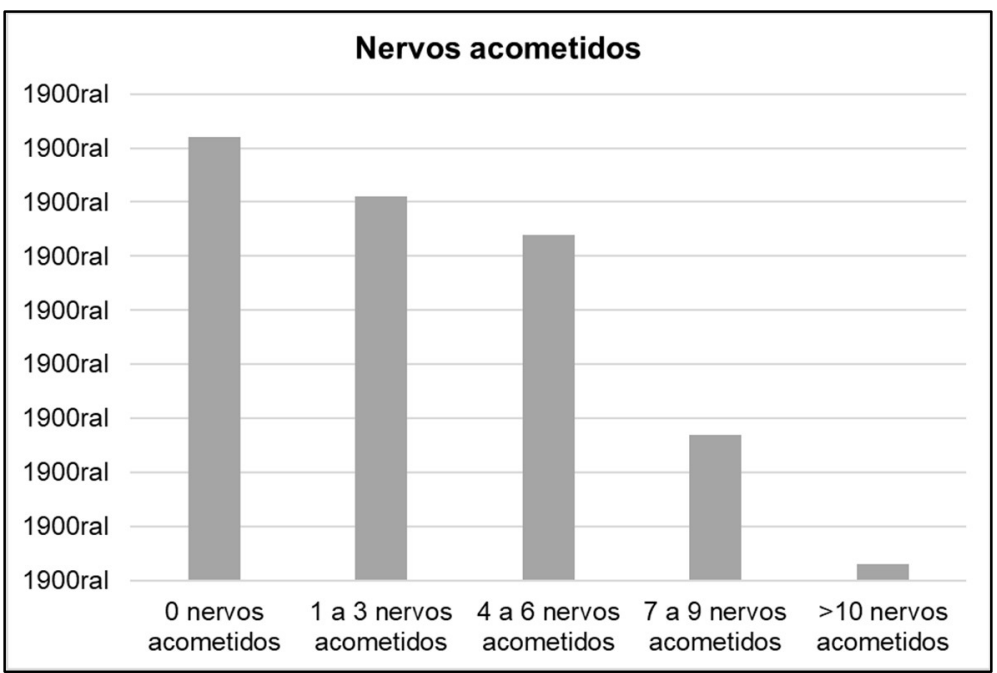

Gráfico 1: Frequência de indivíduos quanto ao número de nervos acometidos. Fonte: Elaborada com dados da Vigilância Epidemiológica de Porto Nacional/TO.

Tabela 3: Distribuição da hanseníase quanto à forma clínica da doença.

\begin{tabular}{lllllll}
\hline Forma Clínica & $\mathbf{2 0 1 3}$ & $\mathbf{2 0 1 4}$ & $\mathbf{2 0 1 5}$ & $\mathbf{2 0 1 6}$ & $\mathbf{2 0 1 7}$ & Total \\
\hline Total & 44 & 59 & 41 & 50 & 53 & 247 \\
\hline Indeterminada & 15 & 18 & 3 & 3 & 11 & 50 \\
\hline Tuberculóide & 1 & 8 & 2 & 2 & 5 & 18 \\
\hline Dimorfa & 21 & 27 & 28 & 38 & 32 & 146 \\
\hline Virchowiana & 5 & 4 & 3 & 3 & 2 & 17 \\
\hline Não Classificada & 2 & 2 & 5 & 4 & 3 & 16
\end{tabular}

Fonte: Elaborada com dados da Vigilância Epidemiológica de Porto Nacional/TO.

Tabela 4: Distribuição da hanseníase quanto o critério operacional.

\begin{tabular}{lllllll}
\hline Classificação & $\mathbf{2 0 1 3}$ & $\mathbf{2 0 1 4}$ & $\mathbf{2 0 1 5}$ & $\mathbf{2 0 1 6}$ & $\mathbf{2 0 1 7}$ & Total \\
\hline Total & 44 & 59 & 41 & 50 & 53 & 247 \\
\hline Paucibacilar & 16 & 26 & 7 & 5 & 15 & 69 \\
\hline Multibacilar & 28 & 33 & 34 & 45 & 38 & 178 \\
\hline
\end{tabular}

Fonte: Elaborada com dados da Vigilância Epidemiológica de Porto Nacional/TO.

Por fim, nesse estudo, foi observado um grande número de recidivas, resultando em 12 casos no total. No ano de 2013 não houve nenhum relato. No ano de 2014 e 2016 houve 3 casos cada um, 2014 apenas uma recidiva e em 2017, 5 casos notificados.

\section{DISCUSSÃO}

A hanseníase é uma doença de curso crônico e insidiosa que acomete, principalmente, agrupamento de pessoas, contato contínuo com o indivíduo infectado e nível de classe social mais baixa (WHITE et al., 2015). No Brasil, os altos níveis endêmicos da doença têm colocado como um dos países com mais casos diagnosticados pela OMS (PEIXOTO et al., 2016). Pelos resultados mostrados e estudados dos dados colhidos, foram identificados 4 (quatro) evidências, sendo estas: (1) houve variação no número de infectados entre os anos estudados, (2) prevalência da forma multibacilar (MB) em relação ao paucibacilar (PB), (3) prevalência do sexo masculino, (4) diagnósticos de hanseníase sem acometimento de troncos nervosos.

Nesse trabalho, pode-se observar o comportamento do curso de infecção da hanseníase na 
população do município de porto Nacional/TO. Com relação a estes números, podemos perceber que houve uma grande variação entre os anos estudados, ficando entre os limites de 41 casos/ano a 59 casos/ano em um município de aproximadamente 52 mil habitantes. O resultado é semelhante a nível de proporção ao trabalho de Monteiro, em que evidenciou uma grande discrepância dos casos notificados entre os anos de estudo no estado de Piauí (MONTEIRO et al., 2017), que variava entre 1194 casos/ano a 1348 casos/ano, no período de 2011 a 2015, em um estado de aproximadamente 3.195 milhões.

Enquanto a nível mundial a hanseníase vem apresentando um decréscimo de casos por ano, em muitas regiões do Brasil há a manutenção da doença circulante, ainda que o MS, juntamente com a OMS, tem programado metas para a erradicação da doença (RIBEIRO et al., 2018). Muitas teorias são pregadas para explicar a ausência de diminuição de casos de hanseníase. Entre elas podemos citar a desigualdade socioeconômica entre os vários territórios brasileiros; dificuldade dos pacientes em continuar o seguimento do tratamento, levando ao abandono do mesmo; dificuldade em diagnosticar, mantendo-os como agentes infectantes por muito tempo; e pouca efetividade da vigilância epidemiológica que visa a eliminação total da doença (FREITAS et al., 2017; RIBEIRO et al., 2018). Essas teorias também podem explicar a razão da semelhança de casos diagnosticados neste trabalho.

A forma MB é caracterizada como a principal forma clínica transmissora da doença. Dentre os casos relatados na literatura, esta é responsável por até $75 \%$ dos casos, por ter um longo período de incubação da bactéria (2 a 10 anos), por apresentar sintomas mais tardios e por serem os maiores transmissores do bacilo (ALBUQUERQUE et al., 2018). Percebe-se que essa é a forma predominante no contexto da hanseníase, o que está de encontro com o resultado deste estudo, no qual 178 casos (equivalente a 72,06\%) são do tipo MB.

Dessa forma, como nos casos de MB há uma maior disseminação da doença, se comparado aos casos de PB, o que favorece um diagnóstico tardio, fica evidente que há uma falha na detecção da doença na saúde primária, resultando numa maior disseminação e infecção dos bacilos nos indivíduos susceptíveis. Além disso, na literatura já mostra que a forma MB é a principal causa de incapacidade física, por meio de lesões neurais (COSTA et al., 2017).

A forma MB á caracterizado com mais de 5 (cinco) lesões dermatológicas, de característica hipocromica, sem pelifinicação e com ausência de sudorese local, o que poderia ser de fácil diagnóstico clínico-dermatológico na saúde pública. Entretanto, por ser insidiosa e sem manifestação clínica importante, os indivíduos contaminados demoram a procurar atendimento médico, fazendo com que a doença se propague no organismo e dissemine entre os indivíduos que estão em íntimo contato (LIRA et al., 2018), o que explica, em partes, a dificuldade em erradicar a doença.

A hanseníase, ainda hoje, é uma patologia que há muito preconceito na sociedade. Homens e mulheres sentem vergonha de dizer que apresentam hanseníase, outros infectados relatam desconforto em relação à aparência e aos defeitos neurais que o bacilo pode causar e outros ainda não se sente confiantes em relatar o caso para a família (GIRÃO NETA et al., 2017). Sendo assim, muitos infectados que iniciam o tratamento não se sentem amparados pela família/amigos, tendo por fim o abandono do tratamento e 
reclusão social.

No quesito de gênero, segundo a distribuição de casos, o sexo masculino foi o principal sexo acometido pela doença, o que condiz com a literatura brasileira, já que a nível mundial as mulheres são mais afetadas. Isso se deve ao contato social maior entre os homens, se comparado às mulheres, por compartilhar mais ambientes fechados e, principalmente, por ter menor preocupação com a saúde, retardando a ajuda médica, retardando o diagnóstico, transmitindo o bacilo por todo esse momento (MONTEIRO et al., 2017). Esse estudo condiz com o levantamento feito pelo MS no período de 2012 a 2016, no qual foram relatados 151.764 novos casos, sendo que destes 55,6\% (84.447) eram homens (RODRIGUES, 2018). Neste trabalho a média foi de $62,34 \%$.

Como já foi explicitado nesse artigo, o diagnóstico da hanseníase é essencialmente clínicodermatológico, análise dos sintomas do paciente e, em raríssimos casos, testes específicos. Na maioria dos casos, apenas as lesões hipocrômicas na derme, associado a um contexto epidemiológico, pode dar o diagnóstico definitivo ao paciente (PEIXOTO et al., 2016). Apesar da hanseníase apresentar lesão dermatológica, ela também pode ser precursora de lesão neural, devido ao tropismo das bactérias por esse sistema, o que não é vista em alguns casos, principalmente as paucibacilares. Os principais nervos acometidos são: nervo auricular, supra-orbicular, ulnar, radial, radial cutâneo, mediano, tibial posterior, fibular e sural.

Dessa forma, muitos casos não são definidos por ausência de espessamento do nervo, dor no nervo acometido, paresia, parestesia e outras manifestações neurológicas, já que o bacilo de Hansen acomete o sistema nervoso periférico (GIRÃO NETA et al., 2017). No que tange a manifestação clínica nervosa tardia, em último caso, há a diminuição da força muscular inervados pelos nervos comprometidos, sendo feito, em muitos casos, o diagnóstico definitivo. Dos casos descritos neste estudo, 33,19\% (82 casos) dos casos notificados não apresentavam quaisquer lesões nervosas, apenas dermatológica, o que difere da literatura, já que o diagnóstico é tardio na maioria dos casos e só ocorre após lesões nervosas (FORTUNA et al., 2018). Este resultado evidencia que no Município de Porto Nacional/TO o diagnóstico tem sido feito de forma rápida, antes mesmo de haver lesão nervosa perceptível, mostrando a capacidade dos médicos.

A recidiva da hanseníase é definida como um novo caso de contaminação da bactéria, desenvolvendo sinais e sintomas em pacientes que realizaram o tratamento com poliquimioterapia. Dentre as variações nas manifestações clínicas podemos destacar: aparecimento de novas lesões hipocrômicas, exacerbações de lesões antigas e/ou lesões de tronco nervoso com ou sem neurites (ALMEIDA et al., 2018). Na América Latina o Brasil é o principal país responsável pelos casos de recidivas da doença. Entre o período de 2004 a 2009 foram relatados 1.602 casos de recidivas, sendo que destes, 1.483 ocorreu só no Brasil. Dentre estes, os estados prevalentes são: Mato Grosso, Acre, Amazonas, São Paulo, Paraná e Santa Catarina (BONA et al., 2015). No estudo deste trabalho, foi visto apenas 12 casos de recidivas dentre os 247 casos, o que é considerado muito, já que pelo MS, os casos de recidiva são raros, além de estarem relacionados mais com a forma MB, num prazo de 5 (cinco) a 10 (dez) anos, do que a forma PB, considerada mais difícil de recidiva, e quando ocorre, aparecem após 1 (um) ano após a cura (DINIZ et al., 2009).

Vários são os motivos que podem explicar a razão das recidivas, como: abandono do tratamento, 
insuficiência terapêutica e convívio com outros indivíduos contaminados pela bactéria que não iniciaram o tratamento com poliquimioterapia (PQT) (ALMEIDA et al., 2018). O tratamento da hanseníase foi iniciado na década de 40, porém só iniciou no Brasil após 1991, quando já era preconizado a PQT instituído pela OMS. $O$ tratamento para a hanseníase é longo, durando cerca de 6 meses a 1 ano associado a várias medicações diárias, o que implica em grandes chances de abandono por parte do paciente (TAVARES et al., 2017). Além disso, pelo menos 1 (uma) vez por mês o indivíduo deve comparecer a Unidade Básica de Saúde para realizar uma tomada da medicação. Dessa forma, a locomoção impede que os pacientes se apresentem na Unidade Básica, não aderindo completamente a PQT, aumentando a chance de não curar e recidivar a hanseníase.

Outro aspecto que pode influenciar nas recidivas é a insuficiência terapêutica, levando a resistência bacteriana. Muitos indivíduos contaminados apresentam fatores que impedem que o princípio ativo do medicamento haja contra a bactéria, como alterações anatômicas no trato gastrointestinal (doença celíaca, gastropatias), ou hiperfunção renal e hepática, acarretando em um metabolismo e excreção aumentados, impedindo a ação total da PQT sobre a bactéria (BONA et al., 2015).

Por fim, muitos indivíduos contaminados que estão realizando tratamento correto convivem com outros indivíduos que também estão infectados, porém estão assintomáticos e sem diagnóstico. Acontece que este é um indivíduo infectante que poderá transmitir o bacilo para outros indivíduos após a cura da doença, acarretando em uma nova infecção. Dessa forma, é importante uma busca ativa de todos os indivíduos que convive com portadores ou pacientes já curados da hanseníase, para que o ciclo de contaminação cesse.

\section{CONCLUSÕES}

Diante do exposto, observou-se que o diagnóstico de hanseníase apresentou variação durante o período de estudo, evidenciando poucas ações de promoção e prevenção no controle da doença. Entretanto, foi visto que o diagnóstico da hanseníase no município de Porto Nacional/TO, é feito de forma rápida, mesmo antes de iniciar sintomas nervosos.

Na forma clínica da doença, pode-se ver predomínio da forma multibacilar, compatível com o que é relatado na literatura. Além do predomínio do sexo masculino sobre o feminino, o que não ocorre a nível mundial. Neste estudo é possível perceber que há muitos casos de recidiva, o que é raro de acordo com o MS. A razão para tal, pode ser explicado pela resistência bacteriana, tratamento insuficiente ou falta de aderência às medicações que causam muitos efeitos colaterais. Este estudo aponta para a necessidade de capacitação e melhorias na busca ativa de indivíduos assintomáticos transmissor da doença, incentivando o diagnóstico e, por fim, a quebra do ciclo da doença.

\section{REFERÊNCIAS}

ALBUQUERQUE, A. M. C.; MOREIRA, J. B. L.; FARIAS, M. L. R.; NOGUEIRA, N. F.; ARAÚJO, A. E.; CARVALHO, L. E.; AMARAL, V. F.; SIMENES NETO, F. R. G.. Análise epidemiológica da hanseníase no município de Reriutaba/Ceará, 2001 a 2006.
Rev. Univ. Vale do Rio Verde, v.16, n.2, 2018. DOI: http://dx.doi.org/10.5892/ruvrd.v16i2.4968.g10951218

ALMEIDA, K. T.; MENEZES, A. M. F.; ALVES, K. A. N.; CARMO FILHO, J. R.; COSTA, A. K. A. N.. Leprosy relapse between the 
years 2005 to 2015. Journal of Nursing, v.12, n.10, p.25282534, 2018.

AMORIM, A. A. S.; PEREIRA, I. S. S. D.; SILVA JUNIOR, E. G.. Análise de qualidade de vida de pacientes acometidos por hanseníase. Rev. Hans., v.1, n.1, 2016.

BONA, S. H.; SILVA, L. O. B. V.; COSTA, U. A.; HOLANDA, A. O. N.; CAMPELO, V.. Recidivas de hanseníase em Centros de Referência de Teresina, Piauí, 2001-2008. Epidemiol. Serv. Saúde, Brasília, v.24, n.4, p.731-738, 2015. DOI: http://dx.doi.org/10.5123/S1679-49742015000400015

COSTA, L. A.; BORDA-PINHEIRO, C. J.; REIS, J. H.; REIS JÚNIOR, S. H.. Análise epidemiológica de hanseníase na microrregião de Tucuruí, Amazônia brasileira, com alto percentual de incapacidade física e de casos entre jovens. Rev. Pan-Amaz. Saúde, v.8, n.3, p.9-17, 2017. DOI: http://dx.doi.org/10.5123/s2176-62232017000300002

DINIZ, L. M.; MOREIRA, M. V.; PUPPIN, M. A.; OLIVEIRA, M. L. W. D. R.. Estudo retrospectiva de recidiva da hanseníase no Estado do Espírito Santo. Rev. da Sociedade Brasileira de Med. Tropical, v.42, n.4, p.420-424, 2009.

FORTUNA, J. A.; CARMO, A. V.; SANTOS, E. P.; SOUZA, H. S.; PEREIRA J. S.; SILVA, L. F.; COSTA, F. M.; BRITO, N. J. N.. Avaliação de prontuários de pacientes com hanseníase de uma Unidade Básica de Saúde em Sinop/MT. Facider Revista Científica, n.11, 2018.

FREITAS, B. H. B. M.; CORTELA, D. C. B.; FERREIRA, S. M. B.. Trend a leprosy in individuals under the age of 15 in Mato Grosso, Brazil, 2001-2013. Rev. Saúde Pública, v.51, n.1, 2017. DOI: http://dx.doi.org/10.1590/S1518$\underline{8787.2017051006884}$

GIRÃO NETA, O. A. G.; ARRUDA, G. M. M. S.; CARVALHO, M. M. B.; GADELHA, R. R. M.. Percepção dos profissionais de saúde e gestores sobre a atenção em hanseníase na estratégia saúde da família. Rev. Bras. Promoção Saúde, v.20, n.2, p.239-248, 2017.

GRACIE, R.; PEIXOTO, J. N. B.; SOARES, F. B. R.; HACKER, M. A. V.-B.. Análise da distribuição geográfica dos casos de hanseníase. Rio de janeiro, 2001 a 2012. Ciênc. Saúde Colet., v.22, n.5, p.35-43, 2017. DOI: https://doi.org/10.1590/1413$\underline{81232017225.24422015}$

LIRA, R. M. N.; SILVA, M. V. S.; GONÇALVES, G. B.. Fatores relacionados ao abandono ou interrupção do tratamento de hanseníase: uma revisão integrativa da literatura. Rev. de Enfermagem da UFPI, v.6, n.4, p.53-58, 2017.
LUNA, C. F.; SANTOS, F. G. B.; TAVARES, C. M.; MEDEIROS, Z. M.; MOREIRA, R. S.. Hanseníase e determinantes sociais de saúde: uma abordagem a partir de métodos quantitativos: Bahia, 2001-2015. Tese (Doutorado em Saúde Pública) Instituto Aggeu Magalhães, Recife, 2018.

MONTALVÃO, L. M.. Diagnóstico e tratamento da hanseníase. Rev. Faipe, v.8, n.1, p.72-84, 2018.

MONTEIRO, L. D.; MARTINS-MELO, F. R.; BRITO, A. L.; LIMA, M. S.; ALENCAR, C. H.; HEUKELBACH, J.. Tendências de hanseníase no Tocantins, um estado hiperendêmico do norte do Brasil, 2001-2012. Cad. Saúde Pública, v.35, n.5, p.971-980, 2015. DOI: http://dx.doi.org/10.1590/0102$\underline{311 \times 00075314}$

MONTEIRO, M. J. S. D.; SANTOS, G. M.; BARRETO, M. T. S.; SILVA, R. V. S.; JESUS, R. L. R.; SILVA, H. J. N.. Perfil epidemiológico de casos de hanseníase em um estado do nordeste brasileiro. Rev. Aten. Saúde, v.15, n.54, p.21-28, 2017. DOI: https://doi.org/10.13037/ras.vol15n54.4766

PEIXOTO, D. L. C.; CARNEIRO, H. M. BRITO, F. I. R.; BARREIRA FILHO, D. M.. Perfil epidemiológico dos casos notificados de hanseníase em uma célula regional de saúde do sertão central cearense. Mostra Cient. Farm., v.3, n.1, 2016.

RIBEIRO, M. D. A.; SILVA, J. C. A.; OLIVEIRA, S. B.. Estudo epidemiológico da hanseníase no Brasil: reflexão sobre as metas de eliminação. Rev. Pan-americana de Salud., v.42, n.1, 2018.

RODRIGUES, A. C.. Hanseníase e suas manifestações clínicas e lesões de pele. Rev. Uniplac, v.6, n.1, p.17-24, 2018.

RODRIGUES, R. S. A.. Aspectos clínicos, epidemiológicos e laboratoriais da hanseníase: revisão. Monografia (Especialização em Ciências Farmacêuticas) - Universidade Federal do Paraná, Curitiba, 2016.

TAVARES, C. M.; TARGINO, S. L.; NASCIMENTO, V. A.; GOMES, N. M. C.; BARROS, P. M. F. P.; GOES, F. S.. Análise do comportamento de recidivas $x$ reações hanseníase em Alagoas. Rev. Port.: Saúde e Socied., v.2, n.2, p.435-445, 2017.

VELOSO, D. S.. Perfil clínico epidemiológico da hanseníase: uma revisão integrativa. Rev. Elet. Acervo Saúde, v.10, n.1, p.1429-1437, 2018.

WHITE, C; PAREDES, C. F.. Leprosy in the $21^{\text {st }}$ century. Clinical Microbiology Reviews, v.1, n.28, p.80-94, 2015.

A CBPC - Companhia Brasileira de Produção Científica (CNPJ: 11.221.422/0001-03) detém os direitos materiais desta publicação. Os direitos referem-se à publicação do trabalho em qualquer parte do mundo, incluindo os direitos às renovações, expansões e disseminações da contribuição, bem como outros direitos subsidiários. Todos os trabalhos publicados eletronicamente poderão posteriormente ser publicados em coletâneas impressas sob coordenação da Sustenere Publishing, da Companhia Brasileira de Produção Científica e seus parceiros autorizados. Os (as) autores (as) preservam os direitos autorais, mas não têm permissão para a publicação da contribuição em outro meio, impresso ou digital, em português ou em tradução. 